\title{
Application of Lagrange Mechanics for Analysis of the Light-Like Particle Motion in Pseudo-Riemann Space
}

\author{
Wladimir Belayev
}

Center for Relativity and Astrophysics, 185 Box, 194358, Saint-Petersburg, Russia

\begin{abstract}
We consider variation of energy of the light-like particle in the pseudo-Riemann space-time, find lagrangian, canonical momenta and forces. Equations of the critical curve are obtained by the nonzero energy integral variation in accordance with principles of the calculus of variations in mechanics. This method is compared with the Fermat's principle for the stationary gravity field. The produced equations are solved for the metrics of Schwarzschild, FLRW model for the flat space and Gödel. For these spaces effective mass of light-like particle is established. Relativistic analogue of inertial mass for photon is determined in central gravity field in empty space.
\end{abstract}

Keywords variational methods, energy integral, light-like particle, canonical momenta and forces, Fermat's principle

\section{Introduction}

One of postulates of general relativity is claim that in gravity field in the absence of other forces the word lines of the material particles and the light rays are geodesics. In differential geometry a geodesic line in case of not null path is defined as a curve, whose tangent vector is parallel propagated along itself[1]. The differential equations of geodesic can be found also by the variation method as a path of extremal length with the aid of the virtual displacements of coordinates $x^{i}$ on a small quantity $\omega^{i}$. When we add variation to coordinate of the material particle, the time-like interval slow changes, though that leaves it time-like.

Finding of the differential equations of the null geodesic, corresponding to the light ray motion, by calculus of variations is described in[2]. In space-time with metrical coefficients $g_{i j}$ it is considered variation of the first integral of these equations

$$
\eta=\mathrm{g}_{\mathrm{ij}} \frac{\mathrm{dx}^{\mathrm{i}}}{\mathrm{d} \mu} \frac{\mathrm{dx}}{\mathrm{d} \mu}
$$

where $\mu$ is affine parameter. Deriving variation for extremum determination we must admit arbitrary small displacements of coordinates. The variation of integral of $\eta$ expanded in multiple Taylor series is written as (see eq2) where $\mu_{0}, \mu_{1}$ are values of the affine parameter in points, which are linked by found geodesic. The sum of terms containing variations $\omega^{\mathrm{i}}, \mathrm{d} \omega^{\mathrm{i}} / \mathrm{d} \mu$ in first power is equated to null. Under condition $\omega^{\mathrm{i}}\left(\mu_{0}\right)=\omega^{\mathrm{i}}\left(\mu_{1}\right)=0$, this leads to the geodesic equations in form

* Corresponding author:

wbelayev@yandex.ru (Wladimir Belayev)

Published online at http://journal.sapub.org/ijtmp

Copyright $(\underset{2}{ } 2012$ Scientific \& Academic Publishing. All Rights Reserved

$$
\frac{d^{2} x^{\lambda}}{d \mu^{2}}+\Gamma_{i j}^{\lambda} \frac{d x^{i}}{d \mu} \frac{d x^{j}}{d \mu}=0
$$

where $\Gamma_{i j}^{\lambda}$ are Christoffel symbols:

$$
\Gamma_{i j}^{\lambda}=\frac{1}{2} g^{\lambda \gamma}\left(g_{i \gamma, j}+g_{j \gamma, i}-g_{i j, \gamma}\right) .
$$

Here a comma denotes partial differentiation.

The other terms of series in (2), containing variations of coordinates and their derivatives by $\mu$ in more high powers or their products and being able to have nonzero values, don't take into account. Thus such method admits violation of the condition $\eta=0$, which means that with certain coordinates variations the interval a prior becomes time-like or space-like. Since this interval accords with the light ray motion, one leads to the Lorentz-invariance violation in locality, namely, anisotropies.

It is shown for the massive particle[5] that a fundamental space-time discreteness need not contradict Lorentz invariance, and causal set's discreteness is in fact locally Lorentz invariant. The possibility of Lorentz symmetry break for the photon in vacuum by effects from the Plank scale is studied in[3,4]. However, experiments[6] show exceptionally high precision of constancy of the light speed confirmed a Lorentz symmetry in locality, and astrophysical tests don't detect isotropic Lorentz violation[4].

In the method of calculus of variations in the large[7] ones are considered as possible paths along the manifold disregarding kind of interval, not as the trajectories of physical particles. This approach exceeds the limits of classical variational principle in mechanics, according as which virtual motions of the system are compared with cinematically possible motions. 


$$
\begin{aligned}
& \delta I=\int_{\mu_{0}}^{\mu_{1}}\left\{\sum_{n=1 \beta_{1}+\ldots+\beta_{4}=n}^{\infty}\left[\frac{1}{\beta_{1} ! \ldots \beta_{4} !} \frac{\partial^{n} \mathbf{g}_{i j}}{\partial^{\beta_{1}} \mathbf{x}^{1} \ldots \partial^{\beta_{4}} \mathbf{x}^{4}} \frac{\mathrm{d} \mathbf{x}^{i}}{\mathrm{~d} \mu} \frac{\mathrm{d} \mathbf{x}^{j}}{\mathrm{~d} \mu}\left(\boldsymbol{\omega}^{1}\right)^{\beta_{1}} \ldots\left(\boldsymbol{\omega}^{4}\right)^{\beta_{4}}\right]+\mathbf{g}_{i j}\left(2 \frac{\mathrm{d} \mathbf{x}^{i}}{\mathrm{~d} \mu} \frac{\mathrm{d} \boldsymbol{\omega}^{j}}{\mathrm{~d} \mu}+\frac{\mathrm{d} \boldsymbol{\omega}^{i}}{\mathrm{~d} \mu} \frac{\mathrm{d} \boldsymbol{\omega}^{j}}{\mathrm{~d} \mu}\right)+\right.
\end{aligned}
$$

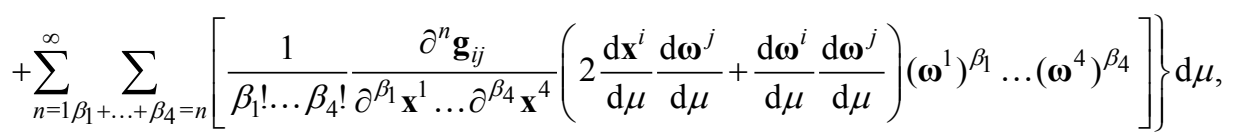

Approximating time-like interval conforming in general relativity to the material particle motion between fixed points to null leads in physical sense to unlimited increase of its momentum, and the space-like interval doesn't conform to move of any object. In this connection it should pay attention on speculation that discreteness at the Planck scale reveals maximum value of momentum for the fundamental particles [8].

The geodesic line must be extremal[1], and the test particle moves along it only in the absence of non-gravity forces. Should photon have some rest mass variations of its path don't give different kinds of intervals, but this assumption doesn't confirm by experiments[9]. We examine choosing of energy so in order that application of variational principle to its integral for deriving of the isotropic critical curves equations would not lead to considering non-null paths.

\section{Definition of Energy and Its Variation}

The interval in pseudo-Riemann space-time with metrical coefficients $\tilde{g}_{\mathrm{ij}}$ :

$$
d s^{2}=\widetilde{g}_{i j} d x^{i} d x^{j}
$$

after substitutions

$$
\widetilde{g}_{11}=\rho^{2} g_{11}, \widetilde{g}_{1 k}=\rho g_{1 k}, \widetilde{g}_{k q}=g_{k q}
$$

is rewritten in form

$$
d s^{2}=\rho^{2} g_{11} d x^{12}+2 \rho g_{1 k} d x^{1} d x^{k}+g_{k q} d x^{k} d x^{q} .
$$

Here, $\rho$ is some quantity, which is assumed to be equal 1 . Putting down $x^{1}$ as time, coordinates with indexes $\mathrm{k}, \mathrm{q}=2,3,4$ as space coordinates and considering $\rho$ as energy of light-like particle with $\mathrm{ds}=0$ [10] we present it as

$$
\rho=\left(g_{11} \frac{d x^{1}}{d \mu}\right)^{-1}\left\{-g_{1 k} \frac{d x^{k}}{d \mu}+\sigma\left[\left(g_{1 k} g_{1 q}-g_{11} g_{k q}\right) \frac{d x^{k}}{d \mu} \frac{d x^{q}}{d \mu}\right]^{1 / 2}\right\},
$$

where $\sigma$ is \pm 1 .

Indexes except $\mathrm{k}, \mathrm{q}$ take values 1 to 4 . With denotation of the velocity four-vector components as $\mathrm{v}^{\mathrm{i}}=\mathrm{dx} \mathrm{x}^{\mathrm{i}} / \mathrm{d} \mu$ energy variation will be

$$
\delta \rho=\frac{\partial \rho}{\partial x^{\lambda}} \delta x^{\lambda}+\frac{\partial \rho}{\partial v^{\lambda}} \delta v^{\lambda}
$$

After substitution

$$
\sigma\left[\left(g_{1 k} g_{1 q}-g_{11} g_{k q}\right) \frac{d x^{k}}{d \mu} \frac{d x^{q}}{d \mu}\right]^{1 / 2}=g_{1 i} \frac{d x^{i}}{d \mu}
$$

the partial derivatives withresect to coordinates are writtens

$$
\frac{\partial \rho}{\partial x^{\lambda}}=\frac{1}{g_{11} v^{1}}\left[-\frac{\partial g_{1 k}}{\partial x^{\lambda}} v^{k}+\frac{1}{2 v_{1}}\left(2 \frac{\partial g_{1 k}}{\partial x^{\lambda}} g_{1 q}-\right.\right.
$$

$$
\left.\left.-\frac{\partial g_{11}}{\partial x^{\lambda}} g_{k q}-\frac{\partial g_{k q}}{\partial x^{\lambda}} g_{11}\right) v^{k} v^{q}\right]-\frac{1}{g_{11}} \frac{\partial g_{11}}{\partial x^{\lambda}} .
$$

This expression is reduced to

$$
\frac{\partial \rho}{\partial x^{\lambda}}=-\frac{1}{2 v_{1} v^{1}} \frac{\partial g_{i j}}{\partial x^{\lambda}} v^{i} v^{j} .
$$

The partial derivatives with respect to components of the velocity four-vector are

$$
\frac{\partial \rho}{\partial v^{\lambda}}=-\frac{v_{\lambda}}{v_{1} v^{1}}
$$

For the particle, moving in empty space, lagrangian is taken in form

and conforms to relation[11]:

$$
L=-\rho,
$$

$$
\rho=v^{\lambda} \frac{\partial L}{\partial v^{\lambda}}-L
$$

which is integral of the motion. Obtained derivatives give the canonical momenta

$$
p_{\lambda}=\frac{\partial L}{\partial v^{\lambda}}=\frac{v_{\lambda}}{v^{1} v_{1}}
$$

and forces

$$
F_{\lambda}=\frac{\partial L}{\partial x^{\lambda}}=\frac{1}{2 v^{1} v_{1}} \frac{\partial g_{i j}}{\partial x^{\lambda}} v^{i} v^{j} .
$$

Components of the associated vector of the canonical momenta are

$$
p^{\lambda}=\frac{v^{\lambda}}{v^{1} v_{1}} .
$$

Units is chosen so that a light velocity constant is $c=1$. Components of energy-momentum four-vector of photon in Minkowski space are proportional to four-velocities: $P^{i}=m_{e f f} v^{i}$ with coefficient of proportionality $m_{e f f}$, which is effective mass. It is represented as

$$
m_{e f f}=h v,
$$

where $h$ is Planck constant and $v$ is frequency of photon. Normalized effective mass of light-like particle in pseudo-Riemannian space-time is defined as coefficient of proportionality between components of the vectors of canonical momenta and the four-velocities with raised indices. It becomes

$$
m_{e f f}^{n}=\frac{1}{v^{1} v_{1}} .
$$

With coefficient of normalization $m_{\text {eff } 0}$ it can be expressed in terms of effective mass as

$$
m_{\text {eff }}^{n}=\frac{m_{\text {eff }}}{m_{\text {eff } 0}} .
$$




\section{Equations of Isotropic Critical Curve}

Motion equations are found from variation of energy integral

$$
S=\int_{\mu_{0}}^{\mu_{1}} \rho d \mu .
$$

Energy $\rho$ is non-zero, its variations leave interval to be light-like, and application of standard variational procedure yields Euler-Lagrange equations

$$
\frac{d}{d \mu} \frac{\partial \rho}{\partial v^{\lambda}}-\frac{\partial \rho}{\partial x^{\lambda}}=0
$$

Critical curve equations are obtained by substitution of partial derivatives (12) and (13) in these equations. For derivative of the first component of four-velocity vector we have

$$
\frac{d v^{1}}{d \mu}+\frac{v^{1}}{2 v_{1}} \frac{\partial g_{i j}}{\partial x^{1}} v^{i} v^{j}=0 .
$$

For finding of other three equations of motion the second term of (23) is presented in form

$$
\begin{gathered}
\frac{d}{d \mu} \frac{\partial \rho}{\partial v^{\lambda}}=\frac{1}{v^{1}\left(v_{1}\right)^{2}}\left[\left(g_{1 k} v_{\lambda}-g_{k \lambda} v_{1}\right) \frac{d v^{k}}{d \mu}-\left(\frac{\partial g_{i \lambda}}{\partial x^{j}} v_{1}-\frac{\partial g_{1 i}}{\partial x^{j}} v_{\lambda}\right) v^{i} v^{j}\right]+ \\
+\frac{g_{11} v^{1} v_{\lambda}+g_{k \lambda} v^{k} v_{1}}{\left(v^{1}\right)^{2} v_{1}^{2}} \frac{d v^{1}}{d \mu} .
\end{gathered}
$$

Replacement of derivative $d v^{1} / d \mu$ here on its expression, obtained from (24), and substitution found terms in EulerLagrange equations gives

$$
\begin{gathered}
\left(g_{k \lambda} v_{1}-g_{1 k} v_{\lambda}\right) \frac{d v^{k}}{d \mu}+\left[\frac{1}{2 v_{1}} \frac{\partial g_{i j}}{\partial x^{1}}\left(g_{11} v^{1} v_{\lambda}+g_{k \lambda} v^{k} v_{1}\right)-\right. \\
\left.-\frac{1}{2} \frac{\partial g_{i j}}{\partial x^{\lambda}} v_{1}+\frac{\partial g_{i \lambda}}{\partial x^{j}} v_{1}-\frac{\partial g_{1 i}}{\partial x^{j}} v_{\lambda}\right] v^{i} v^{j}=0 .
\end{gathered}
$$

These equations contain accelerations corresponded to the space coordinates and describe coupled with (24) the motion of the test light-like particle along critical curve. They don't coincide to usual form (3) of the null geodesics equations.

Let us clear whether proposed variational method conforms to Fermat's principle for stationary gravity field[1], which is formulated as follows

$$
\delta \int \frac{1}{g_{11}}\left(d l+g_{1 k} d x^{k}\right)=0,
$$

where $d l$ is element of spatial distance along the ray

$$
d l^{2}=\left(\frac{g_{1 p} g_{1 q}}{g_{11}}-g_{p q}\right) d x^{p} d x^{q} .
$$

Denoting

$$
d f=\frac{1}{g_{11}}\left(d l+g_{1 k} d x^{k}\right),
$$

and comparing this expression with (8) we write

$$
\frac{d f}{d \mu}=\rho v^{1} \text {. }
$$

Therefore, variation (27) is equivalent to variation of integral

$$
S_{1}=\int_{\mu_{0}}^{\mu_{1}} \rho v^{1} d \mu .
$$

The Euler-Lagrange equation, corresponded to the time coordinate, will be

$$
\frac{\partial \rho}{\partial x^{1}} v^{1}-\frac{d \rho}{d \mu}=0
$$

Since metrical coefficients in case of the stationary field doesn't depend on the time, we have $\partial \rho / \partial x^{1}=0$. The value of $\rho$ is constant. Thus, expression (32) is idential equation.

Equations for the space coordinates are follows:

$$
\frac{\partial \rho}{\partial v^{k}} v^{1}-\frac{d}{d \mu}\left(\frac{\partial \rho}{\partial v^{k}}\right) v^{1}-\frac{\partial \rho}{\partial v^{k}} \frac{d v^{1}}{d \mu}=0
$$

We condition by appropriate choice of the affine parameter $\mu$ the constant value of $v^{1}$. Then, the third term in the left part of equations will be vanishing and they shall be identical to (23).

For the static gravity field the Fermat's principle gives solution, which conforms to geodesics[12].

\section{Photon's Dynamics in Schwarzschild Space-Time}

A centrally symmetric gravity field in the free space is described by the Schwarzschild metric. At spherical coordinates $\mathrm{x}^{\mathrm{i}}=(\mathrm{t}, \mathrm{r}, \theta, \phi)$ its line element is

$$
\begin{aligned}
d s^{2}= & \left(1-\frac{\alpha}{r}\right) d t^{2}-\left(1-\frac{\alpha}{r}\right)^{-1} d r^{2}- \\
& -r^{2}\left(d \theta^{2}+\sin ^{2} \theta d \phi^{2}\right),
\end{aligned}
$$

where $\alpha$ is constant.

For this space we find equations of the critical curve of the integral energy $\rho$. The canonical momenta (16) for the cyclic coordinates $t, \varphi$ are the constants of motion

$$
\begin{gathered}
A=\frac{d t}{d \mu} \\
C=r^{2} \sin ^{2} \theta \frac{d \phi}{d \mu}\left(1-\frac{\alpha}{r}\right)^{-1} .
\end{gathered}
$$

Equations (26) for coordinates $r, \theta$ give

$$
\frac{d^{2} r}{d \mu^{2}}+\frac{\alpha}{2 r^{2}}\left(1-\frac{\alpha}{r}\right)\left(\frac{d t}{d \mu}\right)^{2}-
$$

$$
\begin{array}{r}
-\frac{3 \alpha}{2 r(r-\alpha)}\left(\frac{d r}{d \mu}\right)^{2}-(r-\alpha)\left[\left(\frac{d \theta}{d \mu}\right)^{2}+\sin ^{2} \theta\left(\frac{d \phi}{d \mu}\right)^{2}\right]=0 \\
\frac{d^{2} \theta}{d \mu^{2}}+\frac{2 r-3 \alpha}{r(r-\alpha)} \frac{d r}{d \mu} \frac{d \theta}{d \mu}+\frac{1}{2} \sin 2 \theta\left(\frac{d \phi}{d \mu}\right)^{2}=0
\end{array}
$$

Metric (34) for the isotropic curve yields

$$
\begin{gathered}
\left(1-\frac{\alpha}{r}\right)\left(\frac{d t}{d \mu}\right)^{2}-\left(1-\frac{\alpha}{r}\right)^{-1}\left(\frac{d r}{d \mu}\right)^{2}- \\
-r^{2}\left[\left(\frac{d \theta}{d \mu}\right)^{2}+\sin ^{2} \theta\left(\frac{d \phi}{d \mu}\right)^{2}\right]=0 .
\end{gathered}
$$


Assuming that $A=1$ and considering motion in plane $\theta=\pi / 2$ we write derivatives of the cyclic coordinates

$$
\begin{gathered}
\frac{d t}{d \mu}=1, \\
\frac{d \phi}{d \mu}=\frac{C}{r^{2}}\left(1-\frac{\alpha}{r}\right) .
\end{gathered}
$$

Substituting these values in equation (39) we find

$$
\frac{d r}{d \mu}= \pm\left[\left(1-\frac{\alpha}{r}\right)^{2}-\left(\frac{C}{r}\right)^{2}\left(1-\frac{\alpha}{r}\right)^{3}\right]^{1 / 2} .
$$

Found velocities coincide with solutions of the null geodesic equations for the Schwarzschild space-time[2] to within parameter of differentiation

$$
d \mu=d \mu_{s}\left(1-\frac{\alpha}{r}\right)^{-1},
$$

where $\mu_{s}$ corresponds to geodesic equations.

The canonical momenta (16) and forces (17) are

$$
\begin{gathered}
p_{1}=1, p_{2}=\mp \frac{1}{\left(1-\frac{\alpha}{r}\right)} \sqrt{1-\frac{C^{2}}{r^{2}}\left(1-\frac{\alpha}{r}\right)}, \\
p_{3}=0, p_{4}=-C ; \\
F_{1}=F_{3}=F_{4}=0, \\
F_{2}=\frac{\alpha}{r^{2}\left(1-\frac{\alpha}{r}\right)}-\frac{C^{2}}{r^{3}}+\frac{\alpha C^{2}}{2 r^{4}} .
\end{gathered}
$$

Nonzero components of vector of the canonical momenta with raised indices are

$$
\begin{gathered}
p^{1}=\frac{1}{\left(1-\frac{\alpha}{r}\right)}, \\
p^{2}= \pm \sqrt{1-\frac{C^{2}}{r^{2}}\left(1-\frac{\alpha}{r}\right)}, \\
p^{4}=\frac{C}{r^{2}} .
\end{gathered}
$$

It follows from Eq. (20) that normalized effective mass of photon in the central gravity field changes as

$$
m_{e f f}^{n}=\frac{1}{\left(1-\frac{\alpha}{r}\right)} .
$$

A nonzero component of vector of the canonical forces with raised indices is

$$
F^{2}=-\frac{\alpha}{r^{2}}+\frac{C^{2}}{r^{3}}\left(1-\frac{\alpha}{r}\right)\left(1-\frac{\alpha}{2 r}\right) .
$$

In so far as with gravitational constant $\mathrm{G}$ and mass $\mathrm{M}$ the Newtonian limit of gravity theory requires $\alpha=2 \mathrm{GM}$, the first term of $F^{2}$ yields twice Newton gravity force. One conforms to the light deflection in central gravity field [13], which is twice value being given by the Newton gravity theory.

Substituting components of the velocity four-vector in Eq. (37) we obtain radial the acceleration

$$
\frac{d v^{2}}{d t}=\frac{\alpha}{r^{2}}\left(1-\frac{\alpha}{r}\right)+\frac{C^{2}}{r^{3}}\left(1-\frac{\alpha}{r}\right)^{2}\left(1-\frac{5 \alpha}{2 r}\right) .
$$

A second Newton Law states that massive body under action of the force $\overline{\mathrm{F}}$ experiences acceleration $\overline{\mathrm{a}}=\overline{\mathrm{F}} / \mathrm{m}$, where $m$ is its inertial mass. By analogy normalized inertial mass of photon is found as ratio of the canonical force $\mathrm{F}^{2}$ to the radial acceleration

$$
\begin{aligned}
m_{i n}^{n}= & -\left(1-\frac{\alpha}{r}\right)^{-1}\left\{1-2 C^{2}\left(1-\frac{\alpha}{r}\right)(1-\right. \\
& \left.\left.-\frac{3 \alpha}{2 r}\right)\left[\alpha r+C^{2}\left(1-\frac{\alpha}{r}\right)\left(1-\frac{5 \alpha}{2 r}\right)\right]^{-1}\right\} .
\end{aligned}
$$

With $r \alpha \gg \alpha^{2}, C^{2}$, this yields $m_{\text {in }}^{n} \simeq-m_{\text {eff }}^{n}$. Thus, excepting area, where signs of $\mathrm{F}^{2}$ and $\mathrm{dv}^{2} / \mathrm{dt}$ are identical or one of these quantities is equal to 0 , inertial mass of photon is negative, i. e. it experiences antigravirational influence[14].

\section{Extremal Isotropic Curves in FLRW Space-Time}

The FLRW cosmological model for the flat space with rectangular coordinates $\mathrm{x}^{\mathrm{i}}=\left(\mathrm{t}, \mathrm{x}^{\mathrm{q}}\right)$ is described by metric

$$
d s^{2}=d t^{2}-a^{2}(t) d x^{q 2},
$$

where $a$ is the length scale factor.

Equation (24) gives

$$
\frac{d^{2} t}{d \mu^{2}}-\dot{a} a\left(\frac{d x^{q}}{d \mu}\right)^{2}=0,
$$

where overdot denotes derivative with respect to time. The Euler-Lagrange equations for the cyclic coordinates $x^{q}$ yield constants of motion

$$
p_{q}=-a^{2} \frac{d x^{q}}{d \mu} /\left(\frac{d t}{d \mu}\right)^{2} .
$$

Having extracted derivatives with respect to the space-like coordinates from this equation and substituting them in (52) we obtain

$$
\frac{d^{2} t}{d \mu^{2}}-p_{q}^{2} \frac{\dot{a}}{a^{3}}\left(\frac{d t}{d \mu}\right)^{4}=0 .
$$

This equation has solution, which with denotation $\Pi=p_{q}^{2}$ is written in form

$$
\frac{d t}{d \mu}=\left(\Pi a^{-2}+B\right)^{-1 / 2},
$$

where $B$ is constant. Substitution found first component of the four-velocity vector in equation (53) gives

$$
\frac{d x^{q}}{d \mu}=-p_{q} a^{-2}\left(\Pi a^{-2}+B\right)^{-1} .
$$

The condition, following from Eq. (51):

$$
p_{q}=\left(\frac{d t}{d \mu}\right)^{2}-a^{2}\left(\frac{d x^{q}}{d \mu}\right)^{2},
$$


corresponds to isotropic curve. It yields $\mathrm{B}=0$ and components of the four-velocity vector turn out to

$$
\begin{gathered}
\frac{d t}{d \mu}=\frac{1}{\Pi^{1 / 2}} a, \\
\frac{d x^{q}}{d \mu}=-\frac{p_{q}}{\Pi} .
\end{gathered}
$$

They conform to solution of equations of the null geodesics for the FLRW space-time[2].

The canonical momenta of the light-like particle are

$$
p_{1}=\Pi^{1 / 2} a^{-1}
$$

and constant $p_{q}$. The canonical forces are

$$
F_{1}=\frac{\dot{a}}{a} \Pi, F_{q}=0 .
$$

Their associated values is written as

$$
p^{1}=\Pi^{1 / 2} a^{-1}, p^{q}=-p_{q} a^{-2}
$$

and

$$
F^{1}=\frac{\dot{a}}{a} \Pi, F^{q}=0 .
$$

Normalized effective mass of the photon in the flat space is

$$
m_{\text {eff }}^{n}=\Pi a^{-2} .
$$

Assumed that at present time $t_{0}$ the length scale factor is $\mathrm{a}\left(\mathrm{t}_{0}\right)=1$ and $\mathrm{m}_{\text {eff }}=\mathrm{m}_{\text {effo }}$ we obtain, taking into account Eq. (21), follows:

$$
\Pi=1
$$

and

$$
m_{\text {eff }}^{n}=a^{-2} .
$$

\section{Extremal Isotropic Curves in Gödel Space-Time}

The stationary solution of the Einstein's field equation with cosmological constant found by Gödel describes gravity field of the rotating uniform dust matter. With coordinates $\mathrm{x}^{\mathrm{i}}=(\mathrm{t}, \mathrm{r}, \mathrm{y}, \mathrm{z})$ the line element is written in form

$$
d s^{2}=d t^{2}-d r^{2}-d z^{2}+2 e^{\sqrt{2} \omega r} d t d y+\frac{1}{2} e^{2 \sqrt{2} \omega r} d y^{2},
$$

where $\omega$ is constant.

The canonical momenta (16) for cyclic coordinates $t, y, z$ are the constants of motion. They are written in form

$$
\begin{gathered}
p_{1}=\frac{1}{v^{1}}, \\
p_{3}=\frac{e^{\sqrt{2} \omega r} v^{1}+\frac{1}{2} e^{2 \sqrt{2} \omega r} v^{3}}{v^{1}\left(v^{1}+e^{\sqrt{2} \omega r} v^{3}\right)}, \\
p_{4}=-\frac{v^{4}}{v^{1}\left(v^{1}+e^{\sqrt{2} \omega r} v^{3}\right)} .
\end{gathered}
$$

These equations with following from Eq. (67) condition

$$
0=\left(v^{1}\right)^{2}-\left(v^{2}\right)^{2}-\left(v^{4}\right)^{2}+2 e^{\sqrt{2} \omega r} v^{1} v^{3}+\frac{1}{2} e^{2 \sqrt{2} \omega r}\left(v^{3}\right)^{2}
$$

yield components of the four-velocity vector:

$$
\begin{gathered}
\frac{d t}{d \mu}=\frac{1}{p_{1}}, \\
\frac{d r}{d \mu}= \pm \frac{\left[4 p_{1} p_{3} e^{\sqrt{2} \omega r}-\left(p_{1}^{2}+p_{4}^{2}\right) e^{2 \sqrt{2} \omega r}-2 p_{3}^{2}\right]^{1 / 2}}{p_{1}\left(p_{1} e^{\sqrt{2} \omega r}-2 p_{3}\right)}, \\
\frac{d y}{d \mu}=2 \frac{p_{3}-p_{1} e^{\sqrt{2} \omega r}}{p_{1} e^{\sqrt{2} \omega r}\left(p_{1} e^{\sqrt{2} \omega r}-2 p_{3}\right)}, \\
\frac{d z}{d \mu}=\frac{p_{4} e^{\sqrt{2} \omega r}}{p_{1}\left(p_{1} e^{\sqrt{2} \omega r}-2 p_{3}\right)} .
\end{gathered}
$$

With $p_{1} e^{\sqrt{2} \omega r}=2 p_{3}$ the singularity takes place.

The obtained equations differ from the solution of the null geodesics equations in the Gödel 's space-time[15].

The canonical momentum corresponding to coordinate $r$ is

$$
p_{2}= \pm\left[4 p_{1} p_{3} e^{-\sqrt{2} \omega r}-\left(p_{1}^{2}+p_{4}^{2}\right)-2 p_{3}^{2} e^{-2 \sqrt{2} \omega r}\right]^{1 / 2} .
$$

Canonical forces have values

$$
\begin{gathered}
F_{1}=F_{3}=F_{4}=0, \\
F_{2}=2 \sqrt{2} \omega \frac{p_{3}\left(p_{3}-p_{1} e^{\sqrt{2} \omega r}\right)}{\left(p_{1} e^{\sqrt{2} \omega r}-2 p_{3}\right)^{2}} .
\end{gathered}
$$

Associated canonical momentum and forces are

$$
\begin{gathered}
p^{1}=-p_{1}+2 p_{3} e^{-\sqrt{2} \omega r}, \\
p^{2}=\mp\left[4 p_{1} p_{3} e^{-\sqrt{2} \omega r}-\left(p_{1}^{2}+p_{4}^{2}\right)-2 p_{3}^{2} e^{-2 \sqrt{2} \omega r}\right]^{1 / 2}, \\
p^{3}=2 p_{1} e^{-\sqrt{2} \omega r}-2 p_{3} e^{-2 \sqrt{2} \omega r} \\
p^{4}=-p_{4} ; \\
F^{1}=F^{3}=F^{4}=0, \\
F^{2}=-2 \sqrt{2} \omega \frac{p_{3}\left(p_{3}-p_{1} e^{\sqrt{2} \omega r}\right)}{\left(p_{1} e^{\sqrt{2} \omega r}-2 p_{3}\right)^{2}} .
\end{gathered}
$$

Normalized effective mass of the light-like particle in the Gödel space-time is

$$
m_{e f f}^{n}=\frac{p_{1}}{-p_{1}+2 p_{3} e^{-\sqrt{2} \omega r}} .
$$

\section{Conclusions}

The proposed form of energy allows applying of the Lagrange's mechanics for analysis of the light-like particle motion. Considered procedure of production of the motion equations by variation of the energy integral conforms to principles of the calculus of variations in the classic 
mechanics in accordance with which the motion variations must be cinematically admissible for the system. The virtual displacements of coordinates retain path of the light-like particle to be null in the pseudo-Riemann space-time, i.e. not lead to the Lorentz-invariance violation in locality. For the stationary gravity field the equations, obtained by this method, agrees with result given by the Fermat's principle. Their solutions for the Schwarzschild metric being static and for the Friedmann-Lemaitre-Robertson-Walker metric for the flat space coincide with solutions of the null geodesics equations to within appropriate parameter. For the Gödel 's space-time these solutions are different.

Normalized effective mass of light-like particle is defined as coefficient of proportionality between canonical momenta and components of four-velocity vector. Analog of Newton's inertial mass for photon in Schwarzschild's space-time has negative value for newtonian limit of gravity.

\section{ACKNOWLEDGEMENTS}

I acknowledge J. Foukzon, I. Sivcov and A. Beshenkov for useful discussion.

\section{REFERENCES}

[1] L.D. Landau and E.M. Lifshitz, Classical Theory of Fields, Fourth Revised English Edition, Oxford: Pergamon, 1975.

[2] G.C. McVittie, General Relativity and Cosmology, London, Chapman and Hall Ltd., 1956.

[3] A. Kostelecky and A. Pickering, Vacuum Photon Splitting in Lorentz-Violating Quantum Electrodynamics, Phys. Rev. Lett. 91 (2003) 031801, hep-ph/0212382; T. Jacobson, S. Liberati, D. Mattingly, F.W. Stecker, New limits on Planck scale Lorentz violation in QED, Phys.Rev.Lett. 93 (2004) 021101, astro-ph/0309681.

[4] A. Kostelecky, M. Mewes, Astrophysical Tests of Lorentz and CPT Violation with Photons, Astrophys.J.689 (2008) L1-L4, arXiv:0809.2846.
[5] F. Dowker, J. Henson, R. D. Sorkin, Quantum Gravity Phenomenology, Lorentz Invariance and Discreteness, Mod. Phys.Lett. A19 (2004) 1829-1840, gr-qc/0311055; L. Bombelli, J. Henson, R. D. Sorkin, Discreteness without symmetry breaking: a theorem, gr-qc/0605006.

[6] H. Mueller et al. Relativity tests by complementary rotating Michelson-Morley experiments, Phys.Rev.Lett. 99 (2007) 050401, arXiv: 0706.2031; P. L. Stanwix et al. Improved test of Lorentz Invariance in Electrodynamics using Rotating Cryogenic Sapphire Oscillators, Phys.Rev. D74 (2006) 081101, gr-qc/0609072; Antonini et al. Test of constancy of speed of light with rotating cryogenic optical resonators, Phys. Rev. A. 71 (2005) 050101.

[7] M. Morse, The Calculus of Variations in the Large, Colloquium Publications of the American Mathematical Society, vol. 18. New York, 1934

[8] G. Amelino-Camelia, Doubly Special Relativity, Nature 418 (2002) 34-35, gr-qc/0207049; J. Magueijo and L. Smolin, Lorentz invariance with an invariant energy scale, Phys. Rev. Lett. 88 (2002) 190403, hep-th/0112090.

[9] A.S. Goldhaber and M.M. Nieto, Photon and Graviton Mass Limits, ArXiv:0809.1003.

[10] W.B. Belayev, Variation of the light-like particle energy and its critical curve equations, arXiv:0806.3350.

[11] L.D. Landau and E.M. Lifshitz, Vol. 1. Mechanics, 3ed., Oxford: Pergamon, 1976.

[12] W. Pauli, Theory of relativity, Dover Publications, Inc., 1981.

[13] A. Einstein, Die Grundlage der allgemainen Relativitätstheorie, Ann. Phys. 49 (1916) 769-822.

[14] W.B. Belayev, Effective and Inertial Masses of a Photon Near a Blac Hole for a Family of Envelope Orbits, Wolfram Demonstrations Project, 2011, http://demonstrations.wolfram. com/EffectiveAndInertialMassesOfAPhotonNearABlackHol eForAFamilyO/.

[15] G. Dautcourt, M. Abdel-Megied, Revisiting the Light Cone of the Gödel Universe, Class.Quant.Grav. 23 (2006) 1269-1288, gr-qc/0511015; G. Dautcourt, The lightcone of Gödel-like spacetimes, Class.Quant.Grav.27 (2010) 225024, arXiv: 1009.5231 